PLB Ref. No: 0424

TAUP 2308-95 / solv-int 9512006

revised version

\title{
INTEGRABLE DEFORMATIONS OF THE CFT ON HYPER-ELLIPTIC SURFACES
}

\author{
S. A. Apikyan ${ }^{\dagger}$ \\ Theoretical Physics Department \\ Yerevan Physics Institute \\ Alikhanyan Br.st.2, Yerevan, 375036 ARMENIA \\ C. J. Efthimiou ${ }^{\ddagger}$ \\ Department of Physics and Astronomy \\ Tel-Aviv University \\ Tel Aviv, 69978 ISRAEL
}

\begin{abstract}
In this letter, we continue the work we started at [1] and we propose new series of integrable models in quantum field theory. These models are obtained as perturbed models of the minimal conformal field theories on the hyper-elliptic surfaces by particular relevant operators of the untwisted sector. The quantum group symmetry of the models is also discussed.
\end{abstract}

† e-mail address: apikyan@vx2.yerphi.am

$\ddagger$ e-mail address: costas@ccsg.tau.ac.il 


\section{Introduction}

During the last years the direction of integrable models in mathematical physics has exprerienced a rapid development. In particular, integrable theories from Conformal Field Theory (CFT) have been extremely succesfull due to the revolutionary ideas of A. Zamolodchikov [2]. However, despite of all developments in this area, our knowledge on integrable models cannot be considered as complete as there are still many open problems. For example, the calculation of correlation functions for integrable models is a problem not completely solved up this to this day. Moreover, almost all known integrable models have been constructed on the surface of the sphere; the study of integrable models on higher genus surfaces, although very exciting, has not progressed very much.

Today Riemann surfaces have invaded physics. Among them, the category of hyperelliptic surfaces (HESs) is very special, not only for its simplictity, but also for the fact that it has appeared in many places in theoretical physics. For example, HESs appear in string theory [4], in the Ashkin-Teller model of statistical mechanics [3] etc. One of the most unexpected results was their appearence in the Seiberg-Witten theory of electric-magnetic duality [5].

For the reasons mentioned above, it is therefore quite favorable to explore various aspects and constuctions on HESs. In particular, in this letter, we continue our investigation [1] for constructing integrable models on HESs.

\section{CFT on HESs}

In this short section we review briefly the main aspects of the CFTl on HESs. For a longer discussion, we refer our reader to [6] or to section 2 of [1].

A HES $\Gamma$ is a compact Riemann surface of genus $g \geq 1$ determined by an equation of the form

$$
y^{2}=P_{2 g+2}(z),
$$

where $P_{2 g+2}(z)$ is a polynomial of degree $2 g+2$. $\Gamma$ is therefore a double cover of the sphere and every field $\Phi$ on the sphere, under the hyper-elliptic map (2.1), maps into two fields $\Phi^{(l)}, l=0,1$ living on the two sheets of $\Gamma$.

If $A_{a}, B_{a}, a=1,2, \ldots, g$ denote the basic cycles of the HES, the monodromy operators $\hat{\pi}_{A_{a}}, \hat{\pi}_{B_{a}}$ form a a representation of the $\mathbb{Z}_{2}$ group. Therefore, a CFT built on the HESs will have two sectors: one untwisted (meaning trivial monodromies) and one twisted (meaning non-trivial monodromies). In particular, for the energy momentum tensor in the diagonal basis $T, T^{-}$, we have

$$
\hat{\pi}_{A_{a}} T=T, \quad \hat{\pi}_{A_{a}} T^{-}=T^{-},
$$

\footnotetext{
${ }^{1}$ We should mention here that Krichever and Novikov have also found generalizations of the Virasoro algebra on higher genus surfaces; their work has been discussed and extended by various other authors [7]. Notice that among Riemann surfaces, the HESs are special in the sense that they possess additional $\mathbb{Z}_{2}$ symmetry not present in all surfaces.
} 


$$
\hat{\pi}_{B_{a}} T=T, \quad \hat{\pi}_{B_{a}} T^{-}=T^{-},
$$

in the untwisted sector and

$$
\begin{array}{ll}
\hat{\pi}_{A_{a}} T=T, & \hat{\pi}_{A_{a}} T^{-}=T^{-}, \\
\hat{\pi}_{B_{a}} T=T, & \hat{\pi}_{B_{a}} T^{-}=-T^{-},
\end{array}
$$

in the twisted sector. The corresponding operator product expansions are

$$
\begin{aligned}
T\left(z_{1}\right) T\left(z_{2}\right) & =\frac{c / 2}{z_{12}^{4}}+\frac{2 T\left(z_{2}\right)}{z_{12}^{2}}+\frac{T^{\prime}\left(z_{2}\right)}{z_{12}}+\text { reg } \\
T^{-}\left(z_{1}\right) T^{-}\left(z_{2}\right) & =\frac{c / 2}{z_{12}^{4}}+\frac{2 T\left(z_{2}\right)}{z_{12}^{2}}+\frac{T^{\prime}\left(z_{2}\right)}{z_{12}}+\text { reg } \\
T\left(z_{1}\right) T^{-}\left(z_{2}\right) & =\frac{2}{z_{12}^{2}} T^{-}\left(z_{2}\right)+\frac{T^{\prime}\left(z_{2}\right)}{z_{12}}+\text { reg } .
\end{aligned}
$$

To write the algebra (2.4)-(2.4) in the graded form we determine the mode expansion of $T$ and $T^{-}$:

$$
\begin{aligned}
T(z) V_{(k)}(0) & =\sum_{n \in \mathbb{Z}} z^{n-2} L_{-n} V_{(k)}(0) \\
T^{-}(z) V_{(k)}(0) & =\sum_{n \in \mathbb{Z}} z^{n-2-k / 2} L_{-n+k / 2}^{-} V_{(k)}(0),
\end{aligned}
$$

where $k$ ranges over the values 0,1 and determines the parity sector.

In a Coulomb Gas Formalism, the operators $T, T^{-}$are given by

$$
\begin{aligned}
T & =-\frac{1}{4}\left(\partial_{z} \Phi\right)^{2}+i \alpha_{0} \partial_{z}^{2} \Phi-\frac{1}{4}\left(\partial_{z} \Phi^{-}\right)^{2} \\
T^{-} & =-\frac{1}{2} \partial_{z} \Phi \partial_{z} \Phi^{-}+i \alpha_{0} \partial_{z}^{2} \Phi^{-}
\end{aligned}
$$

where the fields $\Phi, \Phi^{-}$are two free bosons. In analogy with the monodromies of the $T, T^{-}$ fields, in the untwisted sector the bosons have trivial monodromies

$$
\begin{gathered}
\hat{\pi}_{A_{a}} \partial_{z} \Phi=\partial_{z} \Phi, \quad \hat{\pi}_{B_{a}} \partial_{z} \Phi=\partial_{z} \Phi \\
\hat{\pi}_{A_{a}} \partial_{z} \Phi^{-}=\partial_{z} \Phi^{-}, \quad \hat{\pi}_{B_{a}} \partial_{z} \Phi^{-}=\partial_{z} \Phi^{-}
\end{gathered}
$$

while in the twisted sector one of them acquires a minus sign:

$$
\begin{array}{cl}
\hat{\pi}_{A_{a}} \partial_{z} \Phi=\partial_{z} \Phi, & \hat{\pi}_{B_{a}} \partial_{z} \Phi=\partial_{z} \Phi \\
\hat{\pi}_{A_{a}} \partial_{z} \Phi^{-}=\partial_{z} \Phi^{-}, & \hat{\pi}_{B_{a}} \partial_{z} \Phi^{-}=-\partial_{z} \Phi^{-}
\end{array}
$$

In the $k=0$ (untwisted) sector, the vertex operator with charges $\alpha, \beta$ is given by

$$
V_{\alpha \beta}(z)=e^{i \alpha \Phi+i \beta \Phi^{-}}
$$


with conformal weights $\Delta=\alpha^{2}-2 \alpha_{0} \alpha+\beta^{2}$ and $\Delta^{-}=2 \alpha \beta-2 \alpha_{0} \beta$.

In the $k=1$ (twisted) sector there is a twist field $\sigma_{\epsilon}, \epsilon=0,1$ for which

$$
i \partial_{z} \Phi^{-}(z) \sigma_{\epsilon}(0)=\frac{1}{2} z^{-1 / 2} \hat{\sigma}_{\epsilon}(0)+\ldots .
$$

Then, in this sector, the primary fields can be written as

$$
V_{\gamma \epsilon}^{(t)}=e^{i \gamma \Phi} \sigma_{\epsilon}
$$

and have weight $\Delta^{(t)}=\gamma^{2}-2 \alpha_{0} \gamma+1 / 16$.

For the unitary minimal models on the HES the central charge takes the form

$$
c=2-\frac{12}{p(p+1)}, \quad p=3,4, \ldots
$$

The corresponding values of $\alpha, \beta, \gamma$ charges are

$$
\begin{aligned}
& \alpha_{n^{\prime} m^{\prime}}^{n m}=\frac{2-n-n^{\prime}}{2} \alpha_{+}+\frac{2-m-m^{\prime}}{2} \alpha_{-}, \\
& \beta_{n^{\prime} m^{\prime}}^{n m}=\frac{n-n^{\prime}}{2} \alpha_{+}+\frac{m-m^{\prime}}{2} \alpha_{-}, \\
& \gamma_{n m}=\frac{2-n}{2} \alpha_{+}+\frac{2-m}{2} \alpha_{-}, \\
& 1 \leq n, n^{\prime} \leq p, \quad 1 \leq m, m^{\prime} \leq p-1,
\end{aligned}
$$

where the constants $\alpha_{ \pm}$are expressed in terms of the background charge $\alpha_{0}$ :

$$
\alpha_{ \pm}=\alpha_{0} / 2 \pm \sqrt{\alpha_{0}^{2} / 4+1 / 2}
$$

We denote the corresponding fields by $V_{n^{\prime} m^{\prime}}^{n m}, V_{n m}^{(t)}$ and their conformal weights by $\Delta_{n^{\prime} m^{\prime}}^{n m}$, $\Delta_{n m}^{(t)}$. Explicitly

$$
\begin{aligned}
\Delta_{n^{\prime} m^{\prime}}^{n m} & =\frac{\left[\left(n+n^{\prime}\right)(p+1)-\left(m+m^{\prime}\right) p\right]^{2}+\left[\left(n-n^{\prime}\right)(p+1)-\left(m-m^{\prime}\right) p\right]^{2}-4}{8 p(p+1)}, \\
\Delta_{n m}^{(t)} & =\frac{[(p+1) n-m p]^{2}-4}{8 p(p+1)}+\frac{1}{16} .
\end{aligned}
$$

\section{Integrable Deformations of the CFT on the HESs}

In [1], we investigated the integrability of models costructed as deformations of the unitary minimal models $S_{p}\left[\Phi, \Phi^{-}\right]$on HES by primary fields in the twisted sector. In particular, we found that among all series of models

$$
S_{\lambda}[n m ; p]=S_{p}\left[\Phi, \Phi^{-}\right]+\frac{\lambda}{2 \pi} \int d^{2} z V_{n m}^{(t)},
$$


only the series perturbed by $V_{11}^{(t)}$ is integrable:

$$
S_{\lambda}[11 ; p]=S_{p}\left[\Phi, \Phi^{-}\right]+\frac{\lambda}{2 \pi} \int d^{2} z e^{i \gamma_{11} \Phi(z, \bar{z})} \sigma_{\epsilon}(z, \bar{z}) .
$$

The models beloging in this series are all massive and there is no indication of non-trivial fixed points.

Now, we examine deformations of the unitary minimal models on HESs by primary fields in the untwisted sector, i.e. we seek integrable models of the form:

$$
S_{\lambda}\left[\begin{array}{cc}
n & m \\
n^{\prime} & m^{\prime}
\end{array} ; p\right]=S_{p}\left[\Phi, \Phi^{-}\right]+\frac{\lambda}{2 \pi} \int d^{2} z V_{n^{\prime} m^{\prime}}^{n m}(z, \bar{z})
$$

The parameter $\lambda$ is a coupling constant with conformal weights $\left(1-\Delta_{n^{\prime} m^{\prime}}^{n m}, 1-\Delta_{n^{\prime} m^{\prime}}^{n m}\right)$ with respect to $T$.

For a generic perturbation $S_{\lambda}\left[\begin{array}{cc}n & m \\ n^{\prime} & m^{\prime}\end{array} ; p\right]$ cannot be integrable; however, one may be able to choose the primary field $V_{n^{\prime} m^{\prime}}^{n m}(z, \bar{z})$ such that it becomes integrable. To this end, we search for relevant operators for which Zamolochikov's counting argument [8] guarantees the existence of conserved charges. It is not very hard to see that the following models

$$
S_{\lambda}\left[\begin{array}{cc}
1 & 1 \\
n & m
\end{array} ; p\right]=S_{p}\left[\Phi, \Phi^{-}\right]+\frac{\lambda}{2 \pi} \int d^{2} z V_{n m}^{1,1}(z, \bar{z})
$$

where

$$
m=n-1, n, n+1, n+2 .
$$

are almost all integrable. Some comments are in order here:

- 'Almost all models' means that, for some values of $n, m$, there is a minimum number $p_{\min }(m, n)$ such that all models with $p>p_{\min }$ are integrable. When $p \leq p_{\min }$, the perturbations are not relevant.

- As usual, integrability may be spoiled by resonance conditions. However, the phenomenon of resonance conditions is not generic and we will simply interpret the result as 'integrability modulo resonance conditions'.

- Notice that the fields $V_{11}^{n m}, V_{n m}^{11}$ have the same weights relative to $T$ (and opposite weight relative to $T^{-}$). Zamolodchikov's counting argument the arguments is only sensitive to the weights relative to $T$.

In the following, we shall concentrate (for the shake of presentation) on the model with $n=1, m=3$; a similar discussion for the rest models can be also given with few changes. The beta function $\beta(g)$ of the renormalized coupling constant $g=g(\lambda)$ for the first model is given by the equation

$$
\beta_{g}=\varepsilon g-\frac{C}{2} g^{2}+\mathcal{O}\left(g^{3}\right), \quad \varepsilon=1-\Delta_{1,3}^{1,1}=\frac{2}{p+1},
$$


where $C$ is the structure constant

$$
\left\langle V_{1,3}^{1,1}\left(z_{1}, \bar{z}_{1}\right) V_{1,3}^{1,1}\left(z_{2}, \bar{z}_{2}\right) V_{1,3}^{1,1}\left(z_{3}, \bar{z}_{3}\right)\right\rangle=\frac{C}{\left|z_{12}\right|^{2 \Delta_{1,3}^{1,1}}\left|z_{23}\right|^{2 \Delta_{1,3}^{1,1}}\left|z_{31}\right|^{2 \Delta_{1,3}^{1,1}}} .
$$

Depending on the sign of $\lambda$, we have either a massive or a massless theory. In particular, for $\lambda<0$ we do not expect any fixed points and therefore the model is massive; for $\lambda>0$ there is a fixed point and therefore the model flows to a massless theory. We can make this statement more exact by considering large values of $p$. Then one finds

$$
\beta_{g}=g \varepsilon-\frac{2}{\sqrt{3}}\left(1-\frac{3 \varepsilon}{2}\right) g^{2}+\mathcal{O}\left(g^{3}\right) .
$$

When the coupling constant is negative, there is a non-trivial fixed point

$$
g_{*}=\frac{\sqrt{3}}{2} \varepsilon\left(1+\frac{3 \varepsilon}{2}\right)+\mathcal{O}\left(\varepsilon^{3}\right),
$$

which corresponds to the $S_{p-1}\left[\Phi, \Phi^{-}\right]$unitary minimal model of CFT on HES; therefore, in the infrared the theory becomes massless.

\section{The Quantum Group Symmetry of the Models}

The method of non-local conserved charges [9] has been proposed as an alternative to the inverse quantum scattering method. The basic ingredient of the method is the construction of a maximum number of conserved currents

$$
\begin{aligned}
\partial_{\bar{z}} J & =\partial_{z} H, \\
\partial_{z} \bar{J} & =\partial_{\bar{z}} \bar{H},
\end{aligned}
$$

which obey non-trivial braiding relations. These laws lead to the conserved charges

$$
\begin{aligned}
Q & =\int \frac{d z}{2 \pi i} J+\int \frac{d \bar{z}}{2 \pi i} H, \\
\bar{Q} & =\int \frac{d \bar{z}}{2 \pi i} \bar{J}+\int \frac{d z}{2 \pi i} \bar{H},
\end{aligned}
$$

or more compactly

$$
Q=\int_{\mathcal{C}} d z^{\mu} \epsilon_{\mu \nu} J^{\nu}(z)
$$

which act on the Hilbert space $\mathcal{H}=\left\{\Phi^{k}\right\}$

$$
Q\left(\Phi^{k}(w)\right)=\int_{\mathcal{C}(w)} d z^{\mu} \epsilon_{\mu \nu} J^{\nu}(z) \Phi^{k}(w) .
$$

with the following subtlety taken into account: the charge $Q$ is not defined by integrating the currents along an equal time slice, but along a path $\mathcal{C}(w)$ from $-\infty$ to $-\infty$ surrounding the point $w$ of the field and avoiding the cuts on the $z$ plane. 


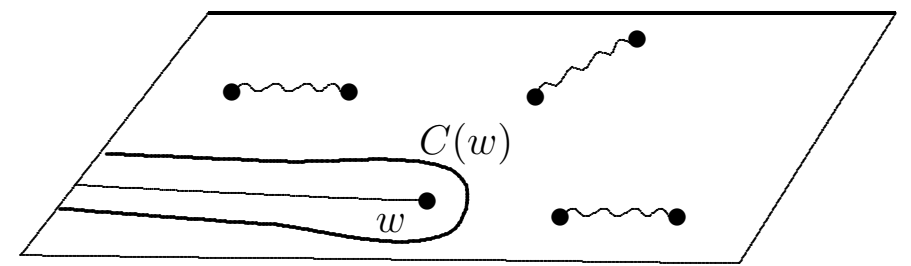

Here, we shall not discuss the way the Hilbert space can be constructed. We point out that such a method of construction has been given in [9, 10, 11] and we refer the interested reader to these papers. The conserved charges constructed as above generate a quantum group which, in turn, determines 2 the S-matrix of the model.

For the action $S_{\lambda}\left[\begin{array}{l}11 \\ 13\end{array}\right]$, one has the following non-local currents:

$$
\begin{aligned}
J_{ \pm}(z) & =e^{ \pm i \frac{1}{2 \alpha_{-}}\left(\varphi(z)+\varphi^{-}(z)\right)} \\
H_{ \pm}(z, \bar{z}) & =\lambda \frac{2 \alpha_{-}^{2}}{2 \alpha_{-}^{2}-1} e^{ \pm i\left(\frac{1}{2 \alpha_{-}}-\alpha_{-}\right)\left(\varphi(z)+\varphi^{-}(z)\right) \mp i \alpha_{-}\left(\bar{\varphi}(\bar{z})+\bar{\varphi}^{-}(\bar{z})\right)} \\
\bar{J}_{ \pm}(\bar{z}) & =e^{\mp i \frac{1}{2 \alpha_{-}}\left(\bar{\varphi}(\bar{z})+\bar{\varphi}^{-}(\bar{z})\right)} \\
\bar{H}_{ \pm}(z, \bar{z}) & =\lambda \frac{2 \alpha_{-}^{2}}{2 \alpha_{-}^{2}-1} e^{\mp i\left(\frac{1}{2 \alpha_{-}}-\alpha_{-}\right)\left(\bar{\varphi}(\bar{z})+\bar{\varphi}^{-}(\bar{z})\right) \pm i \alpha_{-}\left(\varphi(z)+\varphi^{-}(z)\right)}
\end{aligned}
$$

The conserved charges arising from the above currents satisfy the following commutation relations:

$$
\begin{aligned}
& {\left[Q_{+}, \bar{Q}_{+}\right]_{q}=\left[Q_{-}, \bar{Q}_{-}\right]_{q}=0} \\
& {\left[Q_{+}, \bar{Q}_{-}\right]_{q^{-1}}=a\left(1-q^{2 T}\right),} \\
& {\left[Q_{-}, \bar{Q}_{+}\right]_{q^{-1}}=a\left(1-q^{-2 T}\right),}
\end{aligned}
$$

where

$$
[A, B]_{q} \equiv A B-q^{2} B A
$$

and

$$
q=e^{-i \pi / 2 \alpha_{-}^{2}}, \quad a=\frac{\lambda}{2 \pi i}\left(\frac{1}{2 \alpha_{-}^{2}}-1\right) .
$$

Finally, the topological charge is defined by

$$
T=\frac{\alpha_{-}}{2 \pi} \int d x \partial_{x}\left(\Phi+\Phi^{-}\right) .
$$

The algebra defined by the charges $Q_{ \pm}, \bar{Q}_{ \pm}, T$ is a known infinite dimensional algebra, namely the the quantum deformation of the $\mathfrak{s l}(2)$ affine Kac-Moody algebra with vanishing center; the symbol $\widehat{\mathfrak{s l}_{q}(2)}$ is usually used to denote this algebra.

\footnotetext{
${ }^{2}$ Notice that all quantities related with the scattering of particles should be considered on the punctured sphere; in this case the usual LSZ method [13], which requires well defined asymptotic states for the calculation of the S-matrix, is meaningful.
} 
Requiring the S-matrix to commute with the non-local charges, we obtain very stringent constraints

$$
\left[S, \Delta\left(Q_{ \pm}\right)\right]=\left[S, \Delta\left(\bar{Q}_{ \pm}\right)\right]=[S, \Delta(T)]=0
$$

that determine the S-matrix up to a scalar factor [9]. In the above equations, $\Delta(Q)$ denotes the action of the charge $Q$ on an asymptotic pair of solitons.

Before closing the section, we point out that when $n=1, m>1$ the qunatum group structure is similar to the one described above, while in the case $m, n>1$ we have two copies of $\widehat{\mathfrak{s l}_{q}(2)}$.

\section{Discussion}

In this letter we have constructed new integrable models in quantum field theory using deformations of the CFT on HESs by relevant operators. In fact, the CFT on HESs is equivalent to the $W D_{2}$ algebra. Integrable perturbations of the $W D_{n}$ algebras by the relevant operator of scaling dimension $d=\Delta+\bar{\Delta}$, where

$$
\Delta=1-\frac{2(n-1)}{p(p+1)},
$$

have been studied by Lukyanov and Fateev [14]. Moreover, integrable perturbations of the $W$ algebras that generalize the $\Phi_{12}$-perturbed Virasoro models have been considered by Vaysburd and Babichenko [15, 12]. In the above papers the assumption of a simple Lie algebra has been used. However, the underlying algebra of $W D_{2}$ is not simple. In this sense, our discussion completes the discussion of the perturbed $W$ algebras considered by all of the above authors.

\section{Note Added in Proof}

Similar results as the ones reported in section 3 have been obtained independently by I. Vaysburd [16].

\section{References}

[1] S. A. Apikyan and C. Efthimiou, Phys. Lett. B 359 (1995) 313.

[2] G. Mussardo, Phys. Rep. 218 (1992) 215.

[3] Al. B. Zamolodchikov, Nucl. Phys. B 285[FS19] (1987) 481.

[4] V. Knizhnik, Commun. Math. Phys. 112 (1987) 567.

[5] A. Klemm, W. Lerche and S. Yankielowicz, Phys. Lett. B 344 (1995) 169; P. A. Argyres and A. E. Faraggi, Phys. Rev. Lett. 74 (1995) 3931.

[6] C. Crnkovic, G. Sotkov and M. Stanishkov, Phys. Lett. B 220 (1989) 397. 
[7] I. M. Krichever and S. P. Novikov, Funk. Anal. Appl. 21(2) (1987) 46; I. M. Krichever and S. P. Novikov, Funk. Anal. Appl. 21(4) (1987) 47; C. Zachos, L. Mezincescu and R. Nepomechie, Nucl. Phys. B 315 (1989) 43; C. Zachos, L. Mezincescu and R. Nepomechie, Nucl. Phys. B (Proc. Suppl.) 11 (1989) 414; L. Bonora, M. Bregola, P. Cotta-Ramusino and M. Martellini, Phys. Lett. B 205 (1988) 53; L. Bonora, M. Martellini, M. Rinaldi and J. Russo, Phys. Lett. B 206 (1988) 444; D. B. Fairlie, P. Fletcher and J. Nuyts, J. Math. Phys. 30 (1989) 957.

[8] A. B. Zamolodchikov, Int. J. Mod. Phys. A4 (1989) 4235; Adv. Studies in Pure Math. 19 (1989) 641.

[9] D. Bernard and A. LeClair, Commun. Math. Phys. 142 (1991) 99.

[10] G. Felder and A. Leclair, "Restricted Quantum Affine Symmetry of Perturbed Minimal Conformal Models", in RIMS91 Project "Infinite Analysis".

[11] C. J. Efthimiou, Nucl. Phys. B398 (1993) 697.

[12] A. Babichenko, J. Phys. A 28 (1995) 5783.

[13] L. H. Ryder, "Quantum Field Theory", Cambridge University Press 1985.

[14] S. L. Lukyanov and V. A. Fateev, Sov. Phys. Rev. A 15 (1990) 1.

[15] I. Vaysburd, Phys. Let. B 335 (1994) 161.

[16] I. Vaysburd, personal communication; paper in preparation. 